\title{
Accelerated vs. conventional collagen cross-linking for infectious keratitis
}

\author{
ILEANA RAMONA BARAC ${ }^{1 *}$, GEORGE BALTA $^{1}$, MIHAIL ZEMBA $^{1 *}$, \\ LACRAMIOARA BRANDUSE ${ }^{1}$, CLAUDIA MEHEDINTU ${ }^{1}$, MARIAN BURCEA $^{1 *}$, \\ DIANA ANDREEA BARAC ${ }^{1}$, DANIEL CONSTANTIN BRANISTEANU ${ }^{2}$ and FLORIAN BALTA ${ }^{1}$ \\ ${ }^{1}$ Department of Ophthalmology, 'Carol Davila' University of Medicine and Pharmacy, 050474 Bucharest; \\ ${ }^{2}$ Department of Ophthalmology, 'Grigore T. Popa' University of Medicine and Pharmacy, 700115 Iași, Romania
}

Received October 23, 2020; Accepted November 24, 2020

DOI: $10.3892 /$ etm.2021.9716

\begin{abstract}
Infectious keratitis represents a serious concern for ophthalmologists, with a progressively growing incidence in the last few years. In this prospective comparative study, we evaluated two groups of patients with infectious keratitis or corneal ulcer resistant to antimicrobial and antifungal therapy, treated respectively with conventional and accelerated photoactivated chromophore collagen cross-linking. Eight patients were assigned to each group and they were monitored for 12 months. We investigated the differences between groups, comparing on one side the mean of the quantitative variables using the t-test and on the other side the frequencies of qualitative variables using the Fisher exact test. The time to healing for the group treated with conventional cross-linking was 2 days longer than for the group undergoing accelerated cross-linking (34.9 \pm 11.4 vs. $32.9 \pm 9.4$ days), a difference that did not reach statistical significance $(\mathrm{P}=0.708)$. We conclude that the accelerated protocol is as safe and efficient as the classic procedure. The accelerated protocol has an important advantage, both for the doctor and the patient, of being time sparing (the time for accelerated cross-linking is 3 times shorter than in the case of the conventional protocol).
\end{abstract}

\section{Introduction}

Fungal diseases affect over a billion people and kill more than 1.5 million people worldwide (1). Many pathogens become resistant to broad-spectrum antibiotic and antifungal therapy, due to various resistant strains of pathogens. The World Health

Correspondence to: Dr Daniel Constantin Branisteanu, Department of Ophthalmology, 'Grigore T. Popa' University of Medicine and Pharmacy, 16 University Street, 700115 Iasi, Romania E-mail: daniel.branisteanu@umfiasi.ro

*Contributed equally

Key words: collagen cross-linking, infectious keratitis, accelerated photoactivated chromophore collagen cross-linking, corneal ulcer
Organization (WHO) has recently published a list of resistant pathogens involved in infectious keratitis: Methicillin-resistant Staphylococcus aureus (MRSA), vancomycin-intermediate and resistant Staphylococcus aureus, carbapenem-resistant Pseudomonas aeruginosa (PA), and vancomycin-resistant Enterococcus faecium (VRE) (2). Diabetes mellitus, low socioeconomic status, contact lens usage and topical corticosteroids are the main risk factors for fungal keratitis (2).

Infectious keratitis represents a serious concern for ophthalmologists, with a progressively growing incidence in the last few years (3), A recent article published in The Lancet estimates an annual number of 1.4 million cases (4). All of these concerns force us to elaborate on new techniques for better management of these infections Other medical specialties are using alternate treatments for infections management, for example a recent study proves the efficiency of boric acid in vaginal candidosis (5).

Collagen cross-linking (CXL) is a treatment option for corneal ectasia and its application has been recently extended to corneal melting and infectious keratitis. CXL could be a treatment perspective for infectious keratitis resistant to antimicrobial therapy or associated with corneal melting as ultraviolet light and riboflavin have direct antimicrobial effect and increase the mechanical strength of the cornea (6-8). Panda et al (9), Bettis et al (10) and Spoerl et al (11) published important results concerning the possible promising action of CXL for the treatment of infectious keratitis. CXL is thought to have multiple mechanisms of action on the corneal tissue, strengthening the mechanical properties of the cornea, increasing tissue resistance to enzymatic digestion, promoting healing and reducing inflammation, relieving pain and restoring the corneal architecture (7-12). Many recent studies have confirmed the efficacy of the Dresden protocol when using CXL, not only for stopping the progression of keratoconus but also for the treatment of corneal melting and infectious keratitis (12-17). As this protocol is time-consuming, important efforts have been made to decrease the total procedure time. Thus, the so-called 'accelerated protocols' (A-CXL) were developed. Currently, high-intensity UVA lamps, associated with a decrease in irradiation time, allow the same photochemical effect to be obtained. At the 9th International Congress of corneal cross-linking held in 
2013 in Dublin, Ireland, the concept of photoactivated chromophore for infectious keratitis (PACK-CXL) was created in order to better distinguish the use of CXL for the treatment of infectious keratitis from CXL for the treatment of progressive keratoconus (18-20).

In order to investigate the clinical efficacy and safety of accelerated corneal collagen CXL (PACKA-CXL) vs. conventional corneal collagen CXL (PACK-CXL) in the management of infectious keratitis associated with corneal melting, we conducted a prospective comparative study to observe the differences in final visual acuities and healing time between the 2 groups.

\section{Patients and methods}

This prospective, comparative study was approved by the Ethics Committee of Emergency Eye Hospital Bucharest and informed consent was obtained from each patient. Inclusion criteria consisted of adult patients with chronic corneal ulcer with microbial or fungal etiology, hospitalized at the Bucharest Emergency Eye Hospital and Clinic between January 2014 and October 2018. All patients had undergone local and systemic antibiotic and antifungal treatment without any improvement.

Patients were divided into two groups. Group A underwent conventional corneal Collagen CXL (PACK-CXL), using Dresden protocol and group $\mathrm{B}$ underwent accelerated corneal collagen CXL (PACKA-CXL). A complete ophthalmological evaluation including visual acuity, anterior segment slit-lamp evaluation and optical coherence tomography of the cornea was performed at baseline, on first day, postoperatively and at 3 days, 1 week, 2 weeks, 1 month, 2 months, 3 months, 6 months and 12 months, postoperatively.

Direct samplings of the infected corneal ulcer and lower conjunctival fornix were submitted to the central laboratory for culture and antibiogram. The systemic treatment consisted of broad-spectrum new-generation antibiotics and antifungal drugs. The local treatment included fortified antibiotic and antifungal drops and also atropine. Patients with a poor response to medication were included in the study to perform a session of corneal crosslinking. After CXL procedure, the antimicrobial treatment was continued until the healing was complete.

Statistical analysis. The results are presented as the mean and standard deviation for continuous variables, after the previous testing of normal distribution and as absolute and percentage frequencies for the nominal variable. We studied the differences between the 2 groups, comparing on one side the mean of quantitative variables using the t-test and on the other side the frequencies of qualitative variables using the Fisher exact test. We used a statistical significance threshold of 0.05 . The statistical analysis of data was conducted using SPSS 23.0 software (IBM Corp.).

\section{Results}

A total number of 16 eyes from 16 patients were included in the study, 8 eyes randomized for each group. The demographic and clinical parameters of the patients assigned in each group before CXL were similar and are summarized in Table I.
The mean age of the patients from Group A was $39.9 \pm 19.8$ years, not significantly different $(\mathrm{P}=0.967)$ from the mean age of Group B (40.2 \pm 15.0 years). Five patients from Group A and 4 patients from Group B were males, with no significant gender differences between groups $(\mathrm{P}=0.614)$. Five patients from Group A had a central lesion and 3 patients had a paracentral lesion; in Group B the situation was exactly opposite $(\mathrm{P}=0.317)$.

The patients from Group A had previous treatment for $5.2 \pm 2.6$ weeks, not significantly different $(\mathrm{P}=0.738)$ from

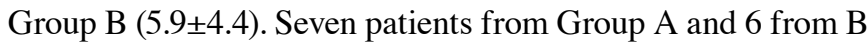
had a positive culture, with no significant differences between groups $(\mathrm{P}=0.522)$.

The ulcer size $\left(\mathrm{mm}^{2}\right)$ and infiltrative area $\left(\mathrm{mm}^{2}\right)$ were not significantly different, but greater in Group A vs. Group B $(17.6 \pm 13.5$ and $29.0 \pm 21.3$ vs. $17.4 \pm 15.7$ and $28.0 \pm 20.1$, respectively). The distribution based on superficial and deep vascularization was not significantly different between the groups $(\mathrm{P}=0.202$ and $\mathrm{P}=0.289$, respectively).

The mean time to healing in patients from Group A (34.9 \pm 11.4 days) was 2 days longer than in Group B (32.9 \pm 9.4 days), but the difference did not reach statistical significance $(\mathrm{P}=0.708)$.

\section{Discussion}

Corneal crosslinking represents an intensely studied method of treatment and many authors have published their results on this subject. Richoz et al conducted a significant and valuable study (16). They inoculated ex vivo porcine corneas with a suspension of Gram-negative (Pseudomonas aeruginosa) and Gram-positive (Staphylococcus aureus) bacterial strains. After incubation, PACK-CXL was performed, at two irradiation settings: $5 \mathrm{~min}$ at $18 \mathrm{~mW} / \mathrm{cm}^{2}$ and $2.5 \mathrm{~min}$ at $36 \mathrm{~mW} / \mathrm{cm}^{2}$. There were significant differences $(\mathrm{P}<0.001)$ between control corneas and cross-linked corneas. The results revealed similar killing rates for both bacterial strains (approximately 93\%), whether they undertook the accelerated or long protocol. The authors concluded that PACK-CXL, when used for treating infectious keratitis, seems to preserve its antimicrobial efficacy, even with shorter time of exposure. After clinical validation, the results can be transferred to routine practice, allowing a shortened treatment time. Tabibian et al reported the case of a 24 -year-old man with fungal keratitis related to contact lens wearing. Accelerated PACK-CXL as a single treatment was performed and evolution of the infiltrate was favorable within $24 \mathrm{~h}$ (17). Makdoumi et al published promising results in 16 patients who underwent PACK-CXL as first-line treatment for infectious keratitis (18). In a prospective study involving 40 patients, 21 treated with PACK-CXL and 19 treated with antimicrobial therapy, Said et al found that the average healing time was shorter in the PACK-CXL group, although not statistically significant (19). In addition, the number of related complications was lower in the PACK-CXL group, supporting the efficacy and safety of PACK-CXL in treating microbial keratitis. Rapuano et al recently described the implications of sodium hydroxymethylglycinate (SMG) in pharmacologic cross-linking of the cornea in the treatment of keratitis (21). The modified crosslinking (M-CXL) 
Table I. Demographic and clinical parameters of groups A and B.

\begin{tabular}{|c|c|c|c|}
\hline Characteristics & Group A (PACK-CXL) $(n=8)$ & Group B (PACKA-CXL) $(n=8)$ & P-value \\
\hline Age, years $($ mean \pm SD) & $39.9 \pm 19.8$ & $40.2 \pm 15.0$ & 0.967 \\
\hline Sex, n (\%) & & & 0.614 \\
\hline Male & $5(62.5)$ & $4(50.0)$ & \\
\hline Female & $3(37.5)$ & $4(50.0)$ & \\
\hline Site & & & 0.317 \\
\hline Central & $5(62.5)$ & $3(37.5)$ & \\
\hline Paracentral & $3(37.5)$ & $5(62.5)$ & \\
\hline Treatment length before presentation (weeks) & $5.2 \pm 2.6$ & $5.9 \pm 4.4$ & 0.738 \\
\hline \multicolumn{4}{|l|}{ Microbiologic culture } \\
\hline Positive & $7(87.5)$ & $6(75.0)$ & 0.522 \\
\hline Ulcer size $\left(\mathrm{mm}^{2}\right)$ & $17.6 \pm 13.5$ & $17.4 \pm 15.7$ & 0.973 \\
\hline Infiltrate area $\left(\mathrm{mm}^{2}\right)$ & $29.0 \pm 21.3$ & $28.0 \pm 20.1$ & 0.924 \\
\hline Time to healing (days) & $34.9 \pm 11.4$ & $32.9 \pm 9.4$ & 0.708 \\
\hline Superficial vascularization & & & 0.202 \\
\hline 0 & $5(62.5)$ & $2(25.0)$ & \\
\hline 1 & $1(12.5)$ & $3(37.5)$ & \\
\hline 2 & $2(25.0)$ & $1(12.5)$ & \\
\hline 3 & $0(0.0)$ & $2(25.0)$ & \\
\hline Deep vascularization & & & 0.289 \\
\hline 0 & $5(62.5)$ & $2(25.0)$ & \\
\hline 1 & $2(25.0)$ & $3(37.5)$ & \\
\hline 2 & $1(12.5)$ & $3(37.5)$ & \\
\hline
\end{tabular}

PACK-CXL, conventional corneal collagen cross-linking; PACKA-CXL, accelerated corneal collagen cross-linking.

performed by Kasparova et al consisted of associating the crosslinking procedure with frequent instillations of antimicrobial drops. In a group of 24 treated patients, they reported a reduction in treatment time by $42 \%$, as compared to the control group treated with conservative treatment (22). Ting et al (20) performed a comprehensive meta-analysis comprising 46 articles and 435 patients. This meta-analysis compared the standard antimicrobial treatment alone for infectious keratitis with PACK-CXL as adjuvant therapy. The authors concluded that the average healing time was less than 7 days for patients treated with adjuvant PACK-CXL when compared to 10 days for standard antimicrobial treatment alone.

In conclusion, our study suggests that the accelerated photoactivated chromophore collagen cross-linking procedure is as safe and efficient as the conventional procedure in infectious keratitis treatment. While having comparable costs, the time required for the accelerated procedure is 3 times shorter thus offering significant advantages for patient comfort.

\section{Acknowledgements}

Not applicable.

\section{Funding}

No funding was received.

\section{Availability of data and materials}

The datasets used and/or analyzed during the current study are available from the corresponding author on reasonable request.

\section{Authors' contributions}

IRB and FB contributed to the study design, participated in the entire review process and prepared the manuscript. GB, LB, $\mathrm{CM}$ and DAB contributed to collecting the relevant literature, data analysis and critical interpretation. DCB, MZ and MB conceived the review concept and modified the manuscript. All authors read and approved the final version of the manuscript.

\section{Ethics approval and consent to participate}

The study was approved by the Ethics Committee of the Emergency Eye Hospital Bucharest (document no. 7/06.01.2020) and informed consent was obtained from each patient.

\section{Patient consent for publication}

Not applicable.

\section{Competing interests}

All the authors declare that they have no competing interests. 


\section{References}

1. Bongomin F, Gago S, Oladele RO and Denning DW: Global and multi-national prevalence of fungal diseases-estimate precision. J Fungi (Basel) 3: 57, 2017.

2. World Health Organization: Global Priority List of Antibiotic Resistant Bacteria to Guide Research, Discovery, and Development of New Antibiotics. Available from: http://www.who.int/medicines/ publications/WHO-PPL-Short_Summary_25Feb-ET_NM_WHO. pdf

3. Acharya Y, Acharya B and Karki P: Fungal keratitis: Study of increasing trend and common determinants. Nepal J Epidemiol 7: 685-693, 2017.

4. Brown L, Leck A, Gichangi M, Burton M and Denning D: The global incidence and diagnosis of fungal keratitis. Lancet 2020.

5. Mehedintu C, Bratila E, Cirstoiu M, Petca A, Brinduse LA, Berceanu C, Barac RI, Andreescu CV, Petca RC, Sandru F, et al: Evaluation of effectiveness and tolerability of boric acid in the treatment of vaginal infection with candida species. Rev Chim 70: 2375-2378, 2019.

6. Neu HC: The crisis in antibiotic resistance. Science 257: 1064-1073, 1992.

7. Chan TC, Lau TW, Lee JW, Wong IY, Jhanji V and Wong RL: Corneal collagen cross-linking for infectious keratitis: An update of clinical studies. Acta Ophthalmol 93: 689-696, 2015.

8. O'Brart DP: Corneal collagen cross-linking: A review. J Optom 7: 113-124, 2013.

9. Panda A, Krishna SN and Kumar S: Photoactivated riboflavin therapy of refractory corneal ulcers. Cornea 31: 1210-1213, 2012.

10. Bettis DI,Hsu M and Moshirfar M: Corneal collagen cross-linking for nonectatic disorders: A systematic review. J Refract Surg 28: 798-807, 2012

11. Spoerl E, Wollensak G and Seiler T: Increased resistance of crosslinked cornea against enzymatic digestion. Curr Eye Res 29: 35-40, 2004.

12. Del Buey MA, Cristóbal JA, Casas P, Goñi P, Clavel A, Mínguez E, Lanchares E, García A and Calvo B: Evaluation of in vitro efficacy of combined riboflavin and ultraviolet a for Acanthamoeba isolates. Am J Ophthalmol 153: 399-404, 2012.

13. Bamdad S, Malekhosseini $\mathrm{H}$ and Khosravi A: Ultraviolet A/riboflavin collagen cross-linking for treatment of moderate bacterial corneal ulcers. Cornea 34: 402-406, 2015.
14. Shetty R, Nagaraja H, Jayadev C, Shivanna Y and Kugar T: Collagen crosslinking in the management of advanced nonresolving microbial keratitis. Br J Ophthalmol 98: 1033-1035, 2014.

15. Ramona BI, Catalina C, Andrei M, Daciana S and Calin T: Collagen crosslinking in the management of microbial keratitis. Rom J Ophthalmol 60: 28-30, 2016.

16. Richoz O, Kling S, Hoogewoud F, Hammer A, Tabibian D, Francois P, Schrenzel J and Hafezi F: Antibacterial efficacy of accelerated photoactivated chromophore for keratitis-corneal collagen cross-linking (PACK-CXL). J Refract Surg 30: 850-854, 2014.

17. Tabibian D, Richoz O, Riat A, Schrenzel J and Hafezi F: Accelerated photoactivated chromophore for keratitis-corneal collagen cross-linking as a first-line and sole treatment in early fungal keratitis. J Refract Surg 30: 855-857, 2014.

18. Makdoumi K, Mortensen J and Crafoord S: Infectious keratitis treated with corneal crosslinking. Cornea 29: 1353-1358, 2010.

19. Said DG, Elalfy MS, Gatzioufas Z, El-Zakzouk ES, Hassan MA, Saif MY, Zaki AA, Dua HS and Hafezi F: Collagen cross-linking with photoactivated riboflavin (PACK-CXL) for the treatment of advanced infectious keratitis with corneal melting. Ophthalmology 121: 1377-1382, 2014.

20. Ting DS, Henein C, Said DG and Dua HS: Photoactivated chromophore for infectious keratitis-corneal cross-linking (PACK-CXL): A systematic review and meta-analysis. Ocul Surf 17: 624-634, 2019.

21. Rapuano P, Scanameo AH, Amponin DE, Paulose SA, Zyablitskaya M, Takaoka A, Suh LH, Nagasaki T, Trokel SL and Paik DC: Antimicrobial studies using the therapeutic tissue cross-linking agent, sodium hydroxymethylglycinate: Implication for treating infectious keratitis. Invest Ophthalmol Vis Sci 59: 332-337, 2018.

22. Kasparova EA, Fedorov AA and Yang B: Clinical results of modified crosslinking in the treatment of purulent keratitis and corneal ulcers. Vestn Oftalmol 136: 64-73, 2020 (In Russian).

This work is licensed under a Creative Commons Attribution-NonCommercial-NoDerivatives 4.0 International (CC BY-NC-ND 4.0) License. 